\title{
Synthesis, Reaction, and Recycle of Fluorous Palladium Catalysts for an Asymmetric Allylic Alkylation without Using Fluorous Solvents
}

Takashi Mino,* Yutaka Sato, Akio Saito, Youichi Tanaka, Hiroaki Saotome, Masami Sakamoto, and Tsutomu Fujita.

Department of Applied Chemistry and Biotechnology, Faculty of Engineering, Chiba University, 1-33, Yayoi-cho, Inage-ku, Chiba 263-8522, Japan

tmino@faculty.chiba-u.jp 


\section{Table of Contents}

Figure S1 _ _ P

Figure S2

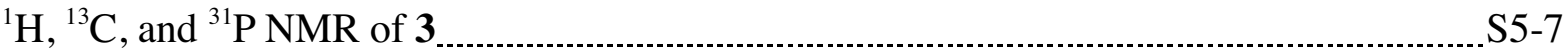

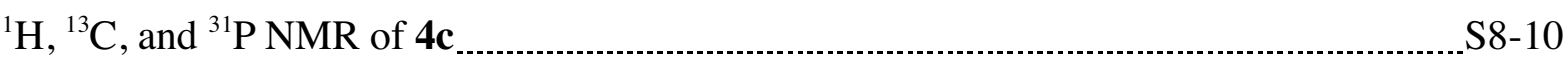

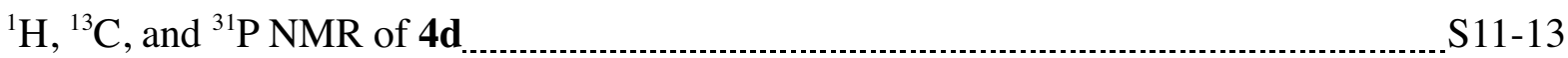

Chiral phase HPLC chart of 9a

Chiral phase HPLC chart of $\mathbf{9 b} \ldots \ldots \ldots \ldots$

Chiral phase HPLC chart of $\mathbf{9 c} \ldots \ldots$ 


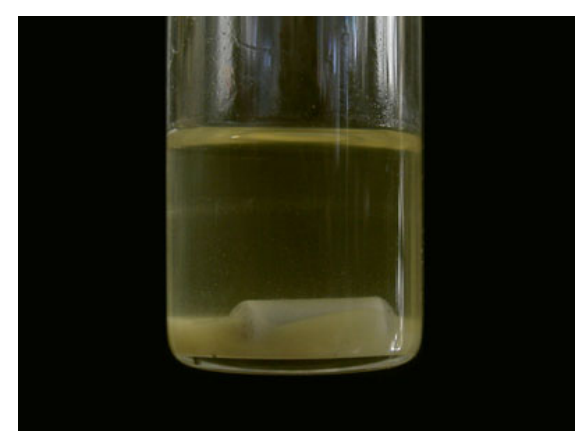

A

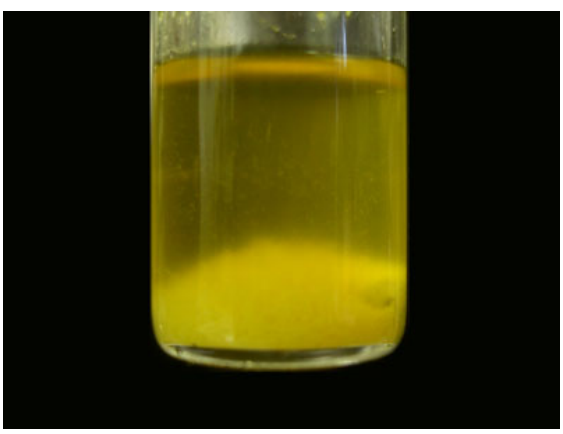

C

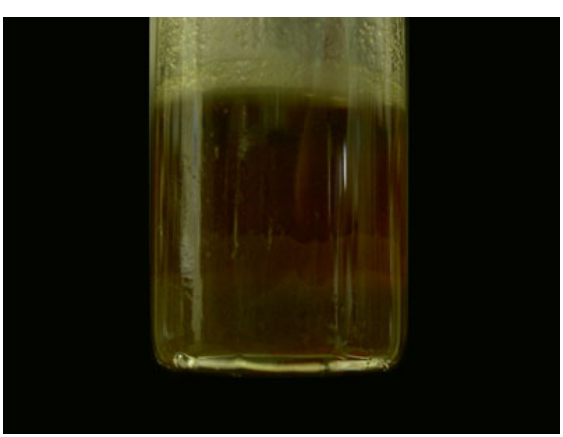

$\mathbf{E}$

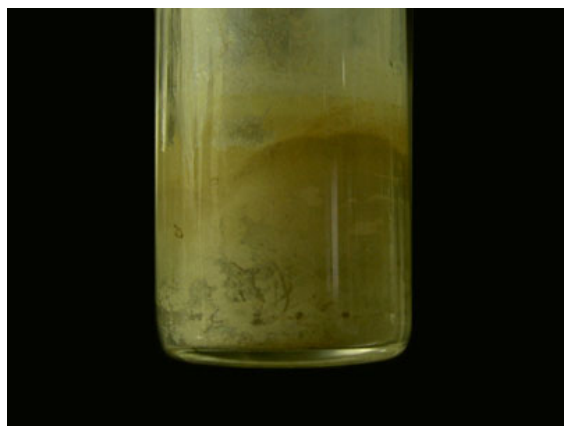

G

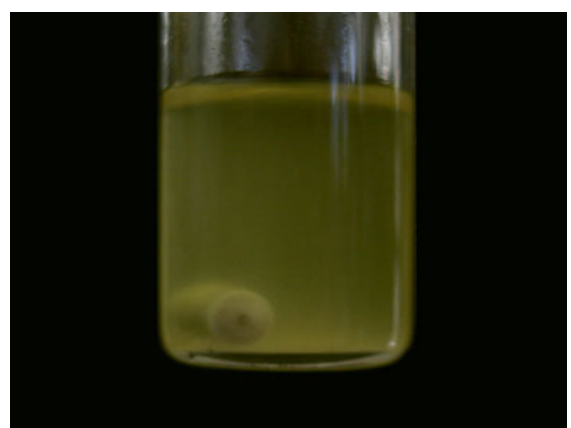

B

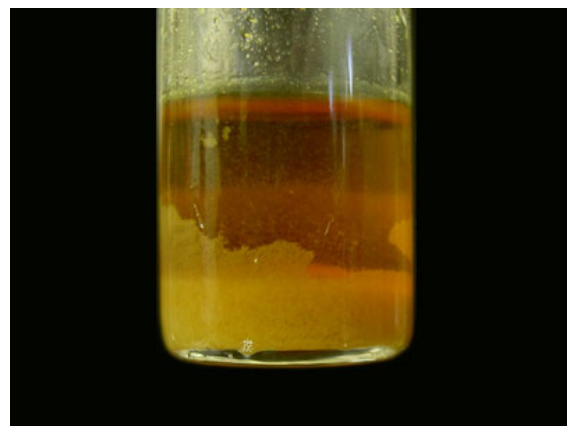

D

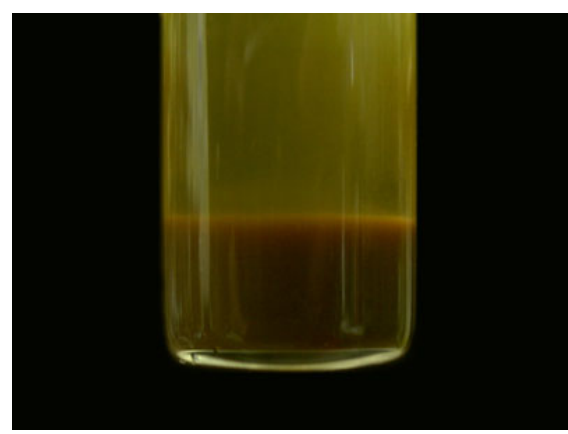

$\mathbf{F}$

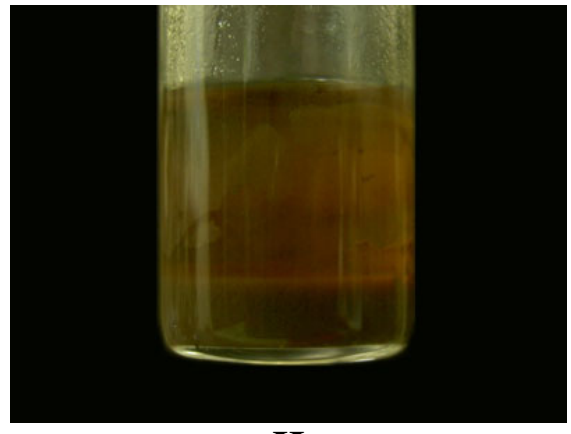

$\mathbf{H}$

Figure S1. Photographs of Fluorous Palladium Complex (entry 6, Table 3): (A) room temperature at start of reaction; (B) $30{ }^{\circ} \mathrm{C}$ at start of reaction; (C) after $1 \mathrm{~h}$; (D) after $2 \mathrm{~h}$; (E) room temperature after reaction; $(\mathrm{F})$ after addition of hexane at $0{ }^{\circ} \mathrm{C} ;(\mathrm{G})$ recovered catalyst; $(\mathrm{H})$ recovered catalyst in diethyl ether. 


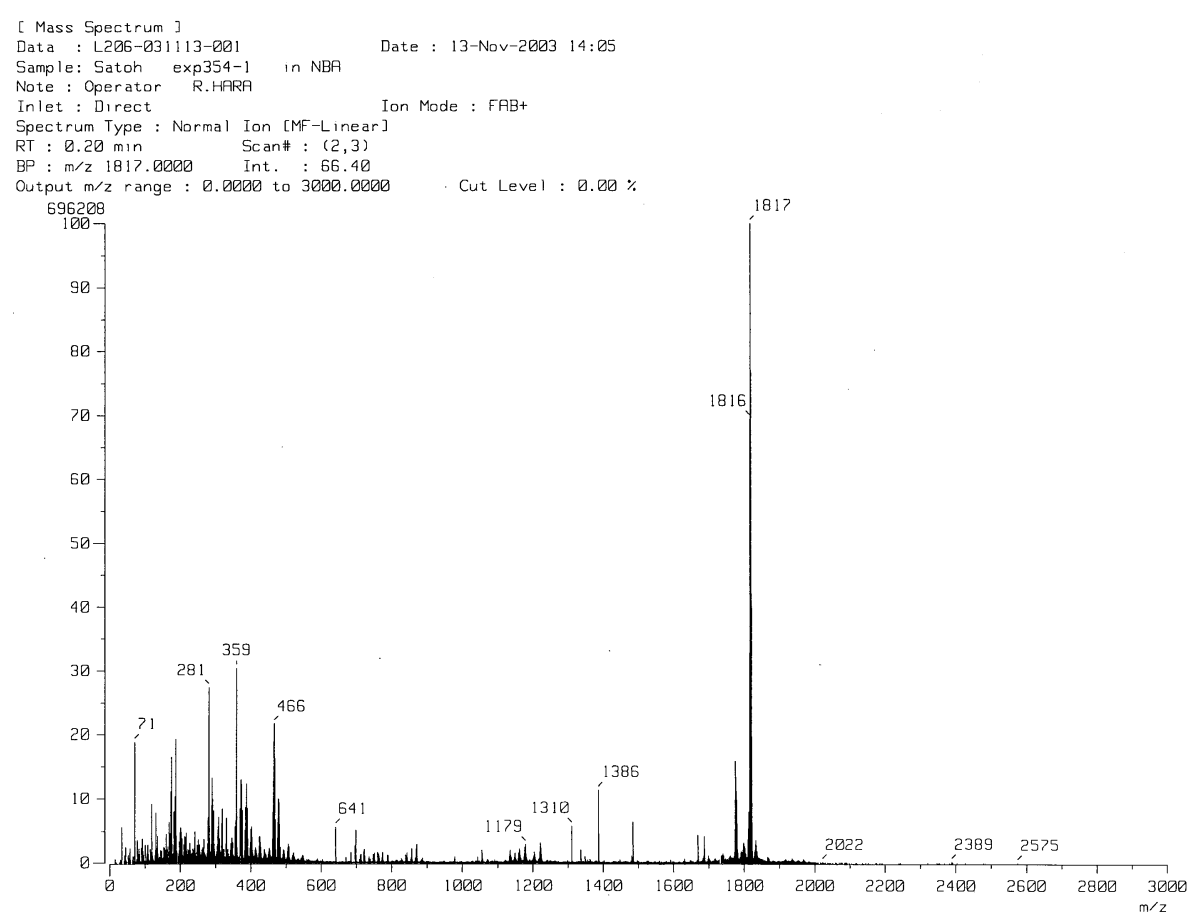

A

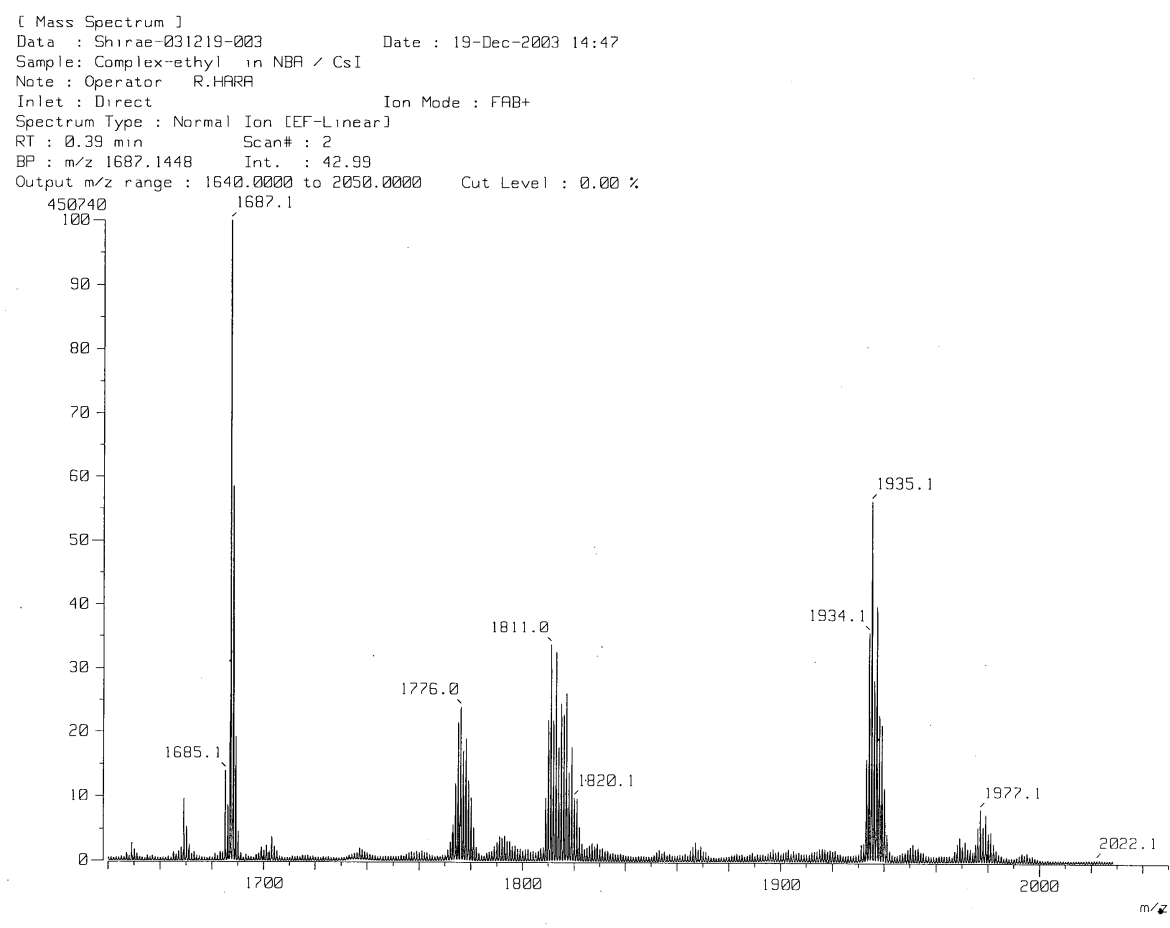

B

Figure S2. The FAB-MS spectrum of palladium complexes: (A) palladium catalyst prepared from $\left[\mathrm{Pd}\left(\eta^{3}-\mathrm{C}_{3} \mathrm{H}_{5}\right) \mathrm{Cl}\right]_{2}$ and ligand $4 \mathbf{c} ;(B)$ recovered palladium catalyst. 


\section{${ }^{1} \mathrm{H}$ NMR of 3}

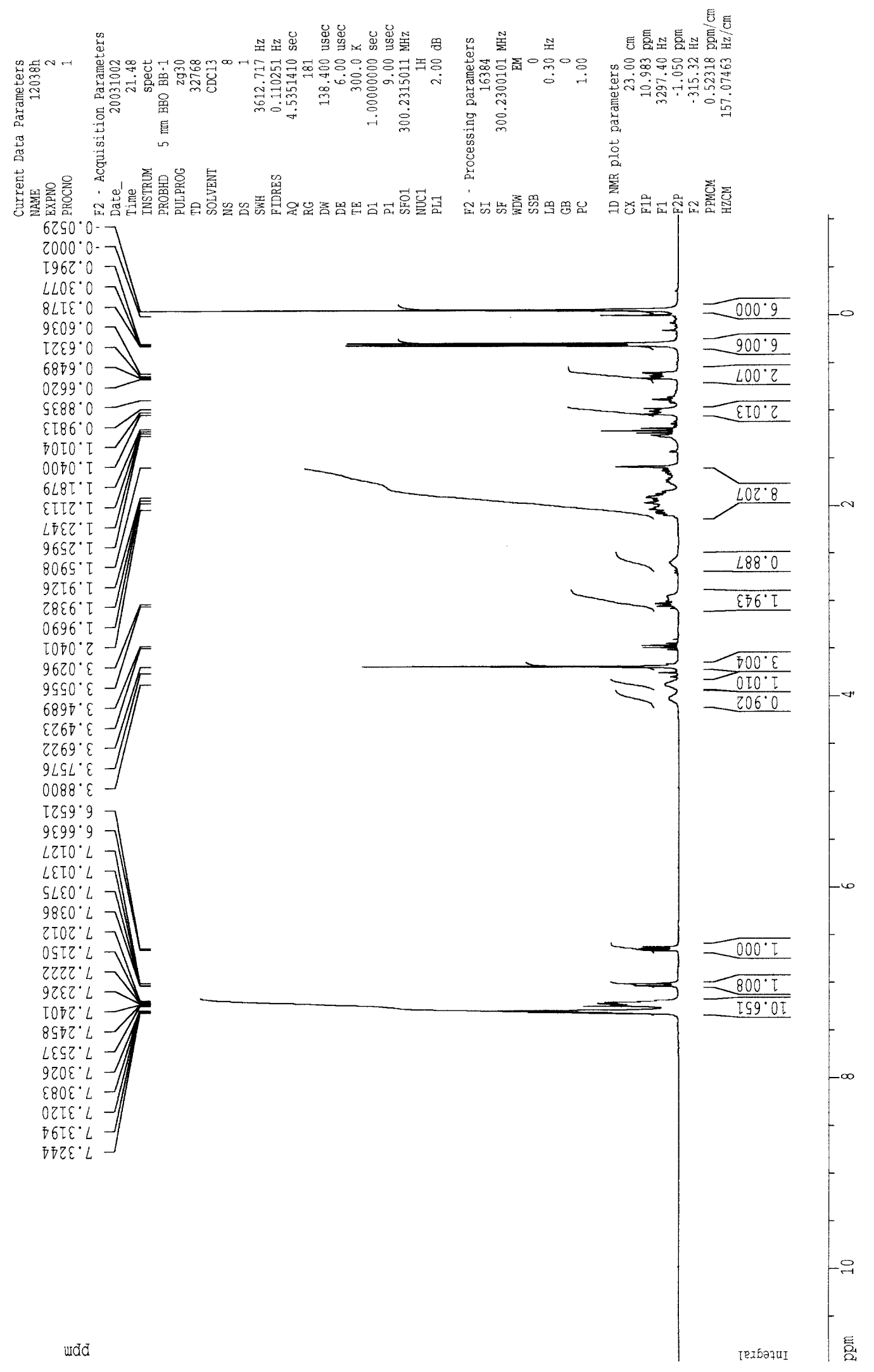


${ }^{13} \mathrm{C}$ NMR of 3
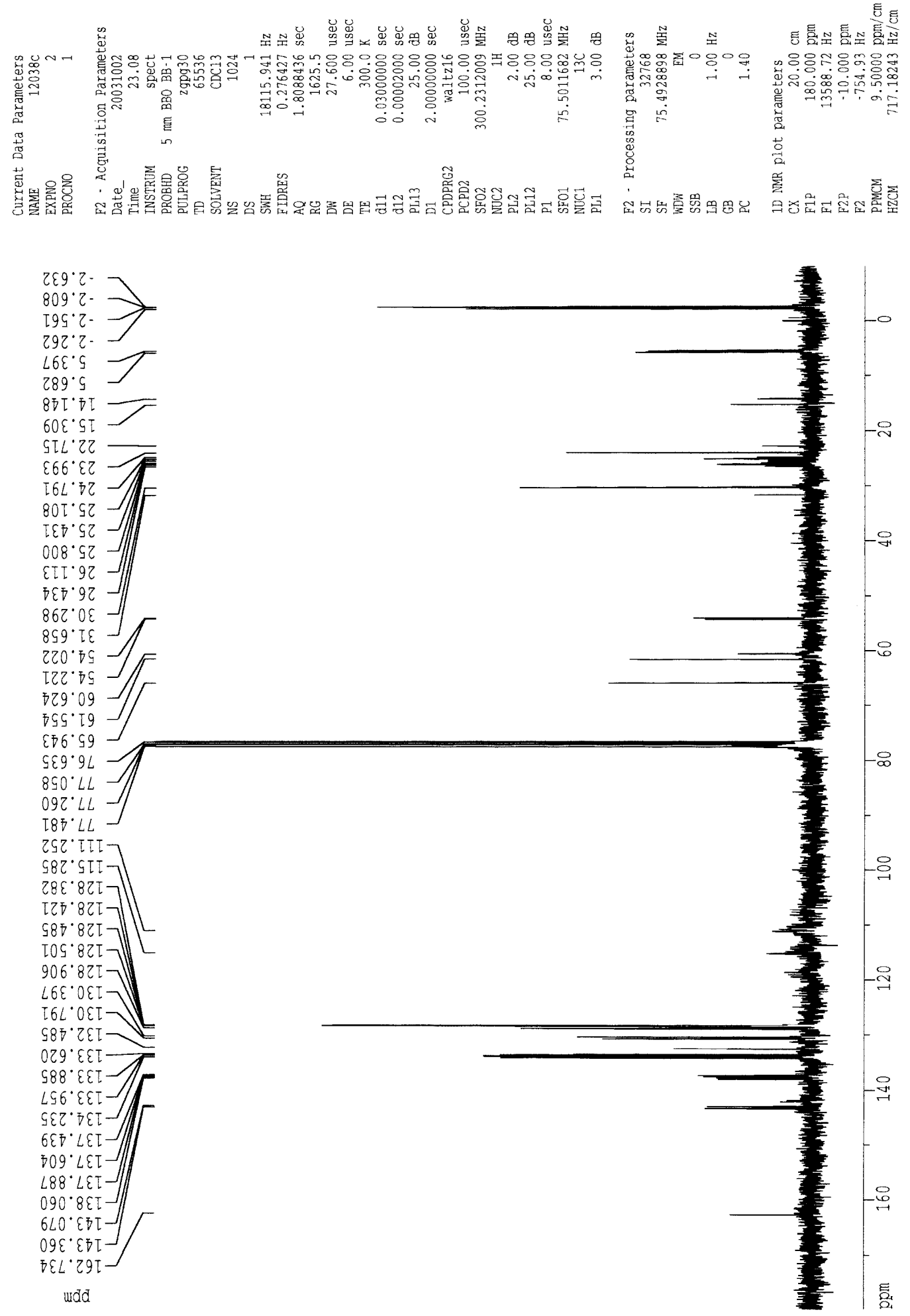
${ }^{31} \mathrm{P}$ NMR of $\mathbf{3}$

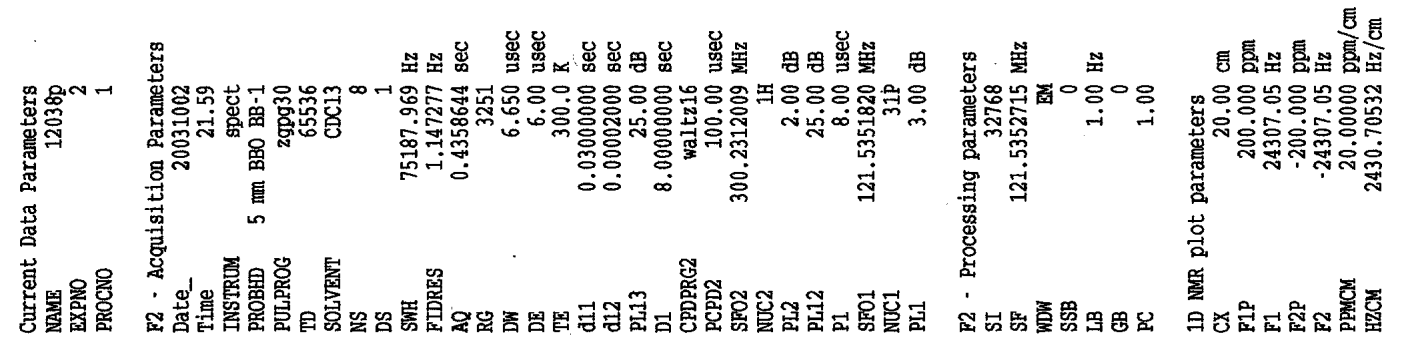

$\tau \tau 9^{\circ} \varepsilon \tau-$

urdd

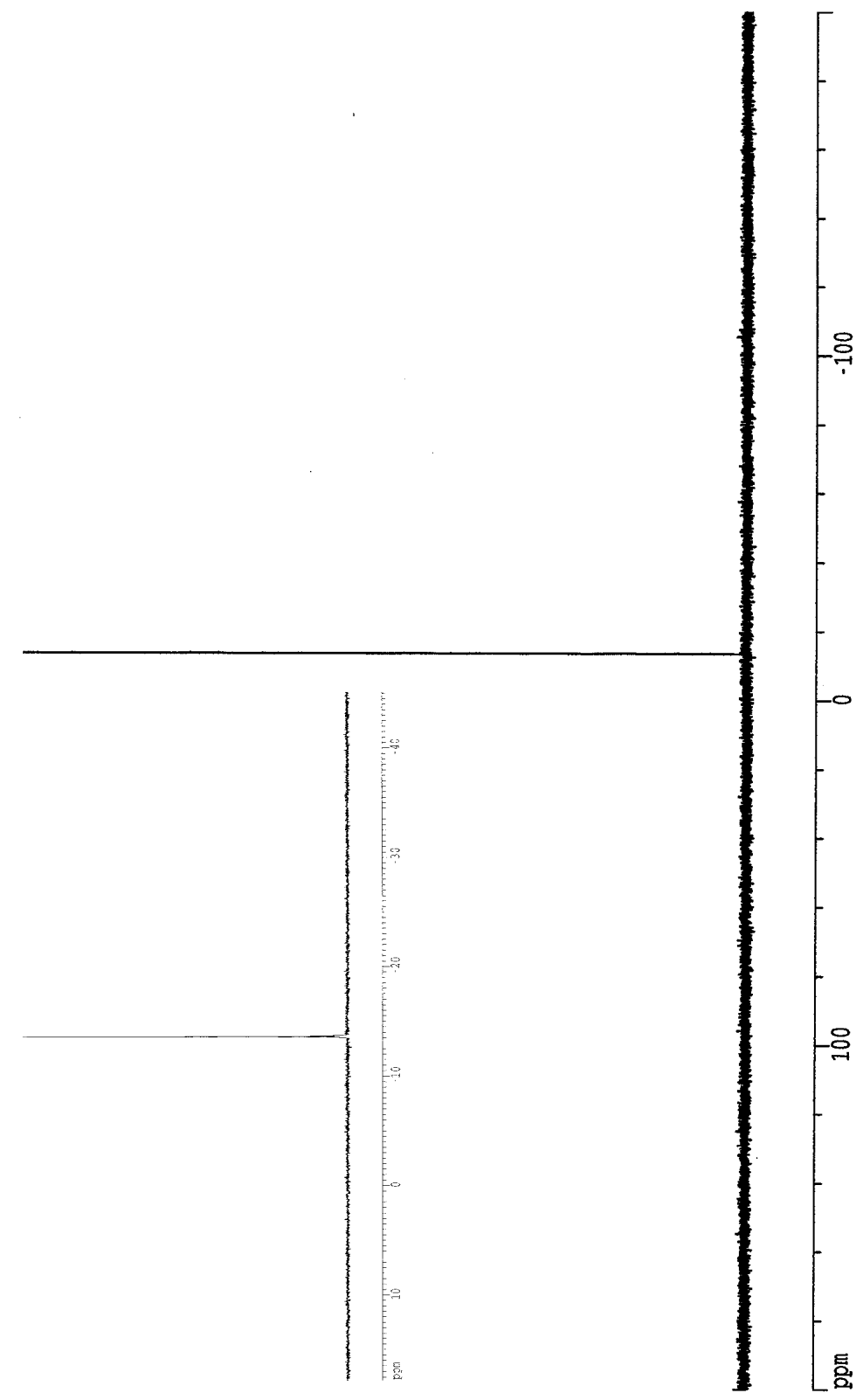


${ }^{1} \mathrm{H}$ NMR of $\mathbf{4 c}$

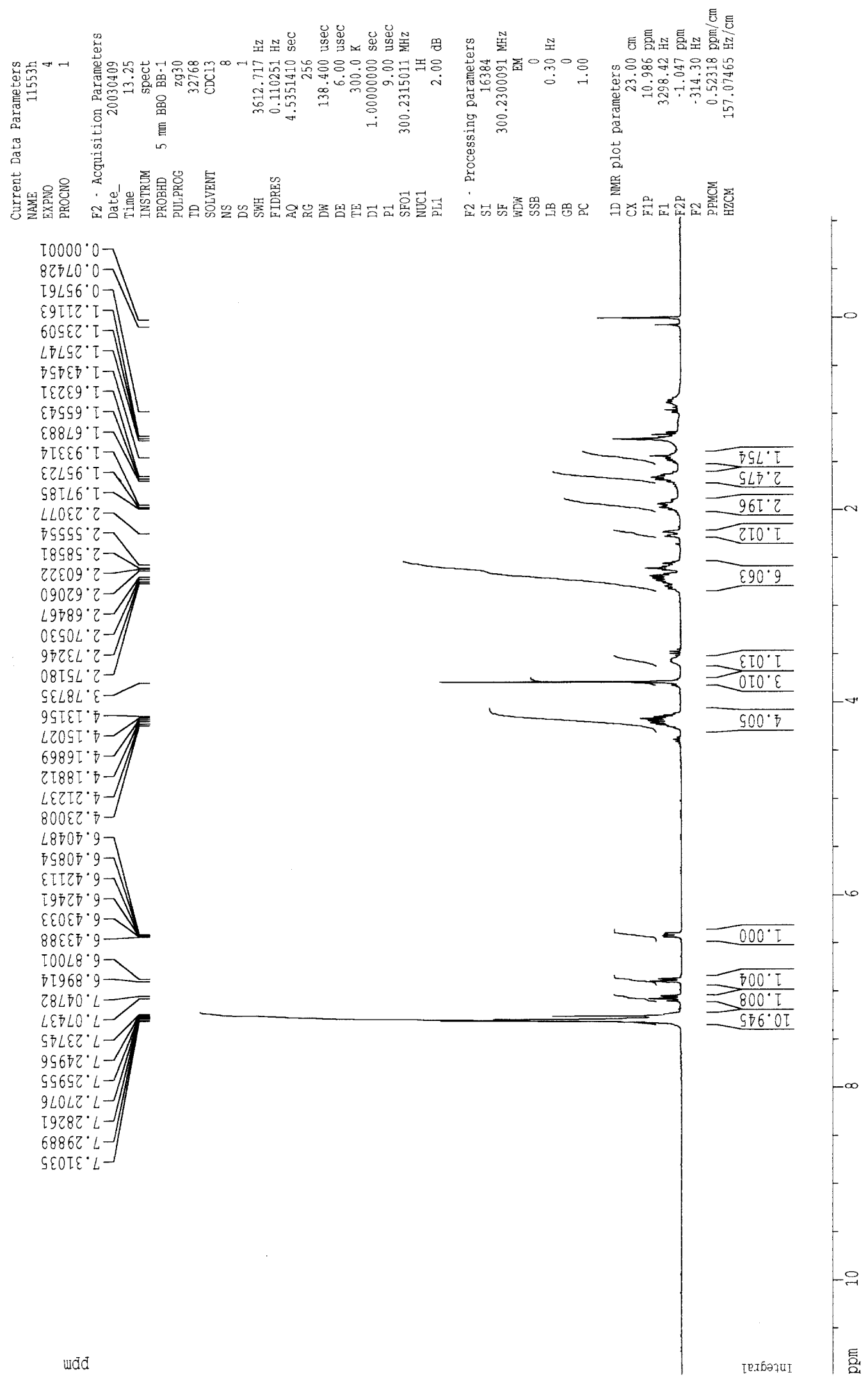



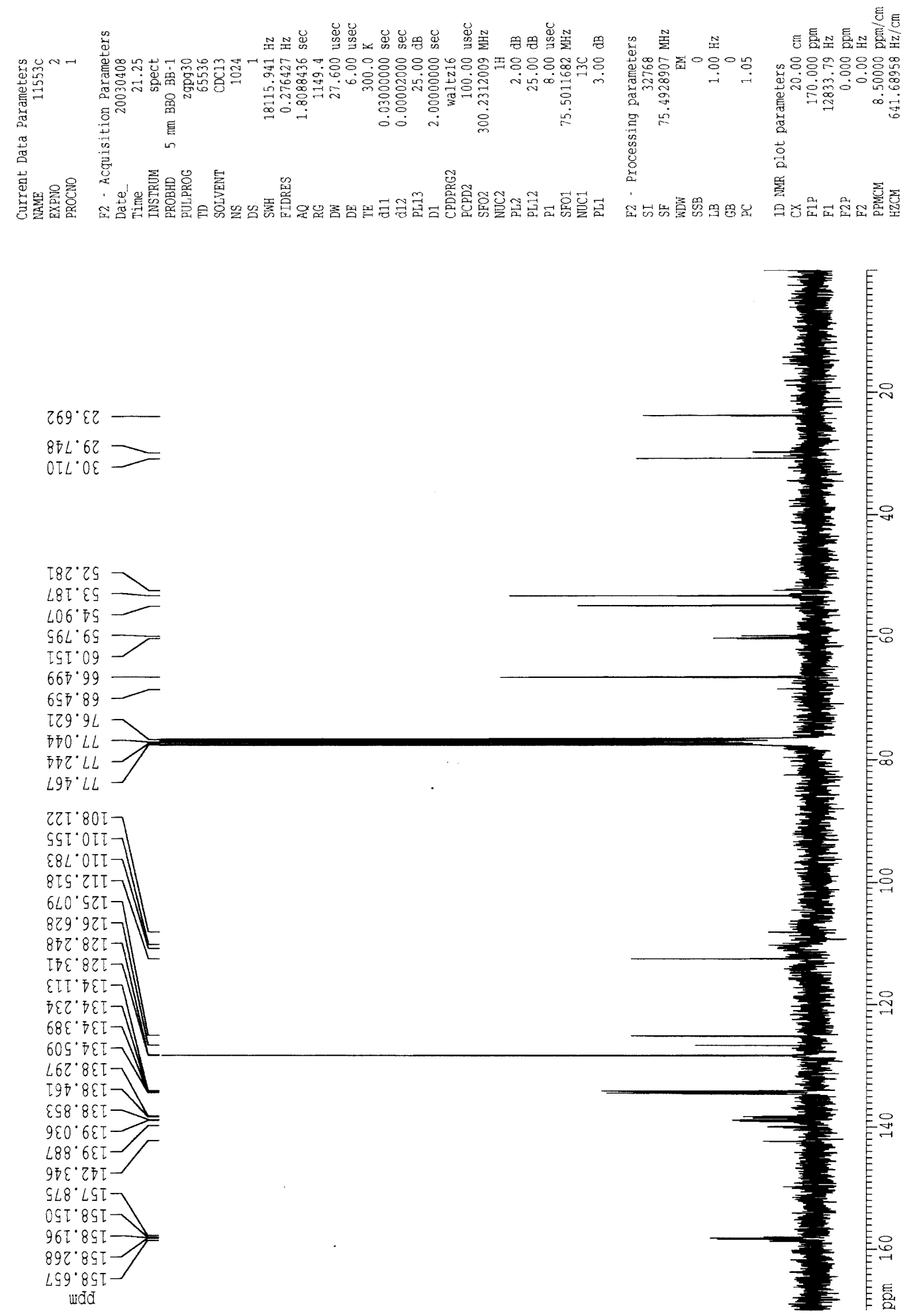
${ }^{31} \mathrm{P}$ NMR of $\mathbf{4 c}$

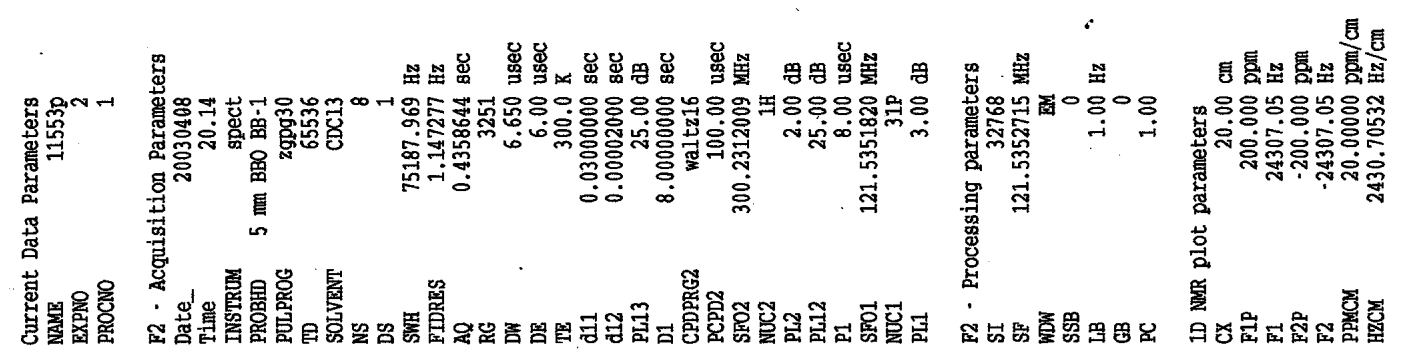

$588^{\circ} 5 \tau-$

แđđ̄

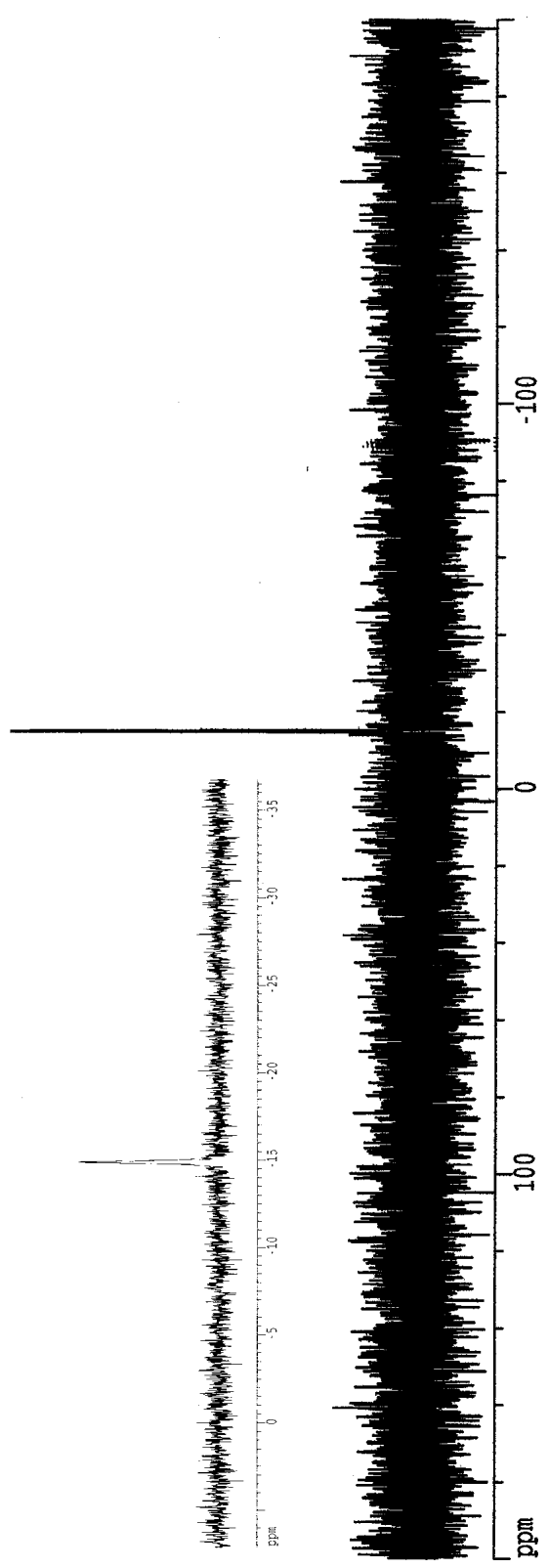




\section{${ }^{1} \mathrm{H}$ NMR of $\mathbf{4 d}$}

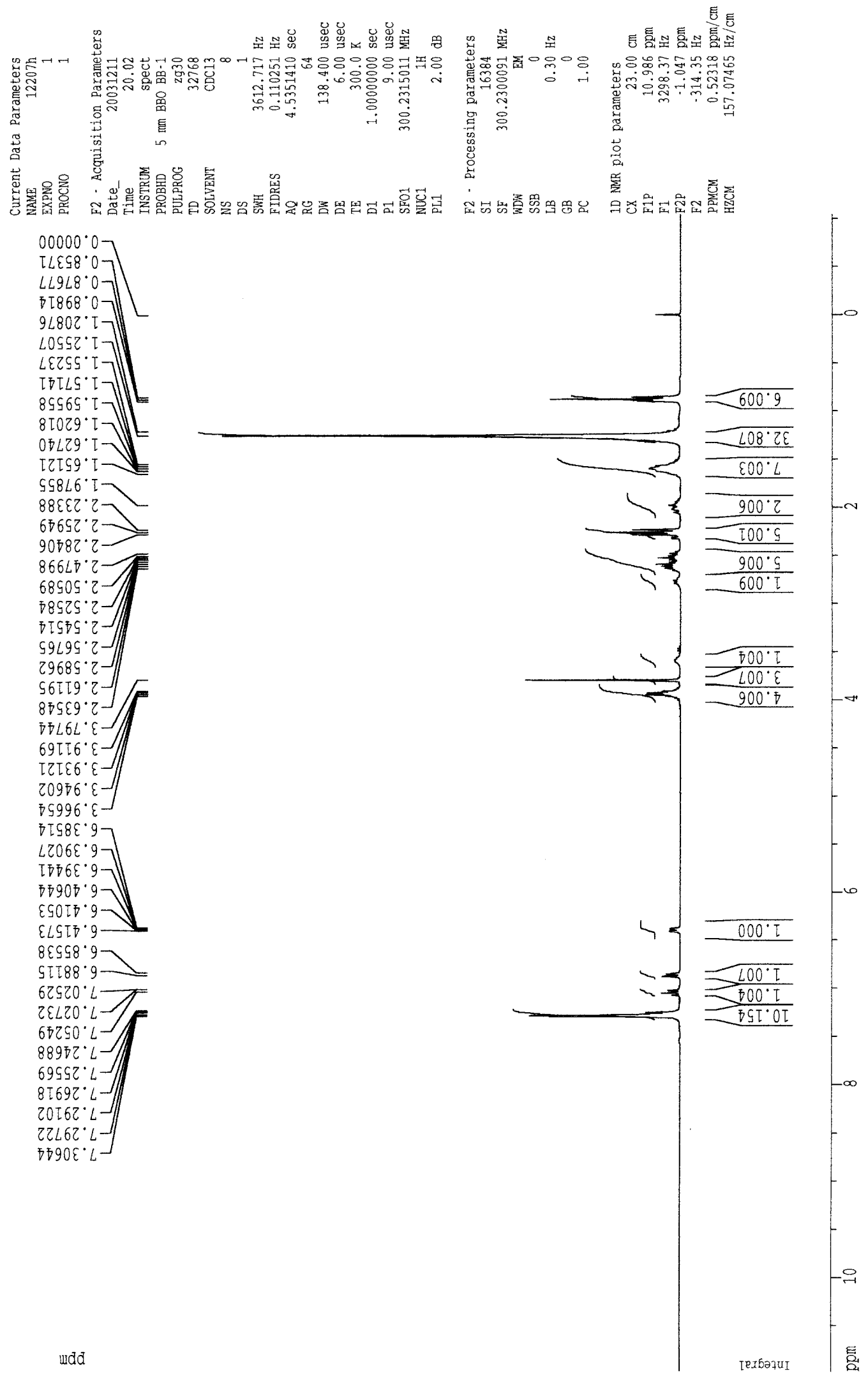



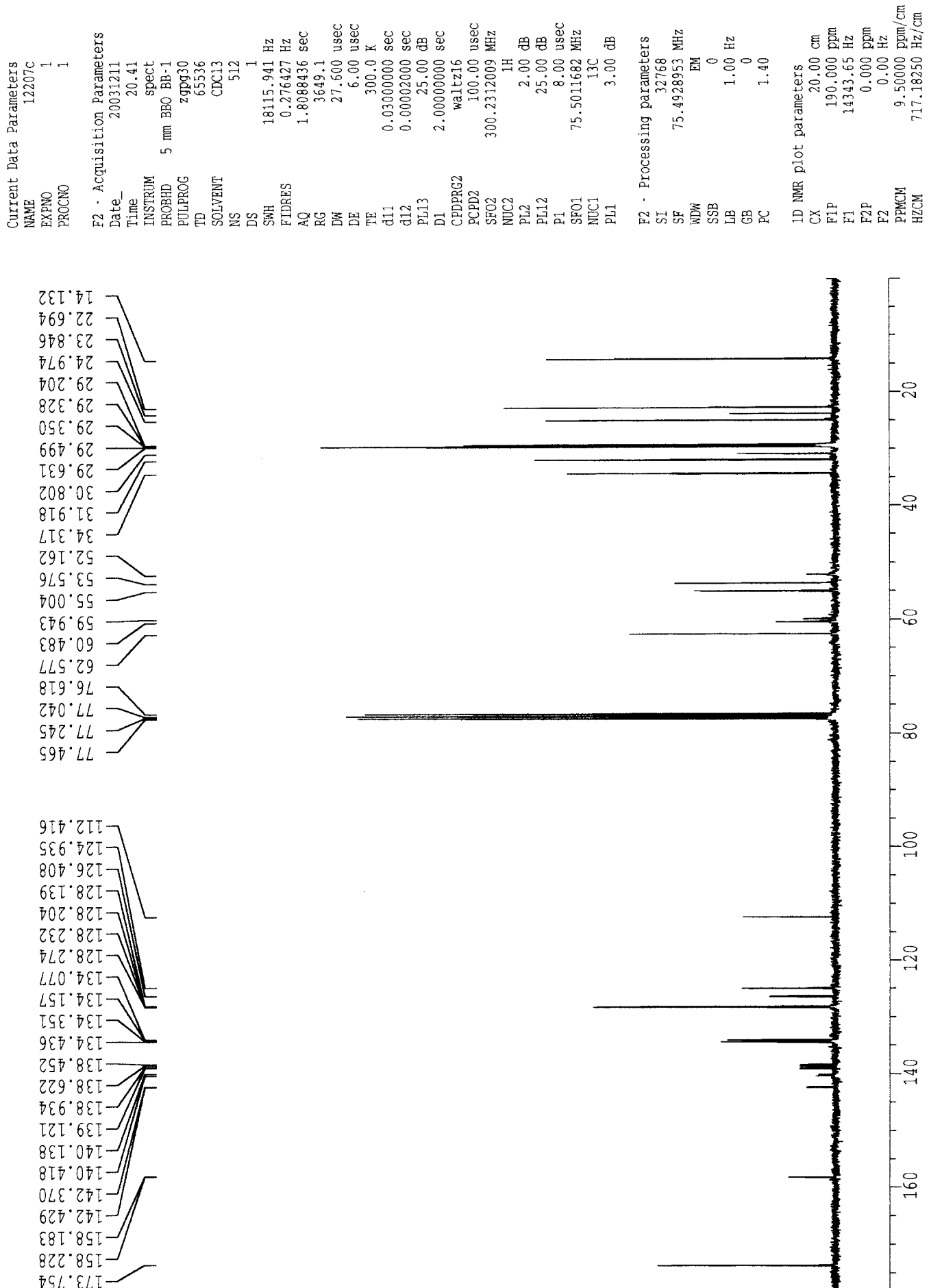

wdđd

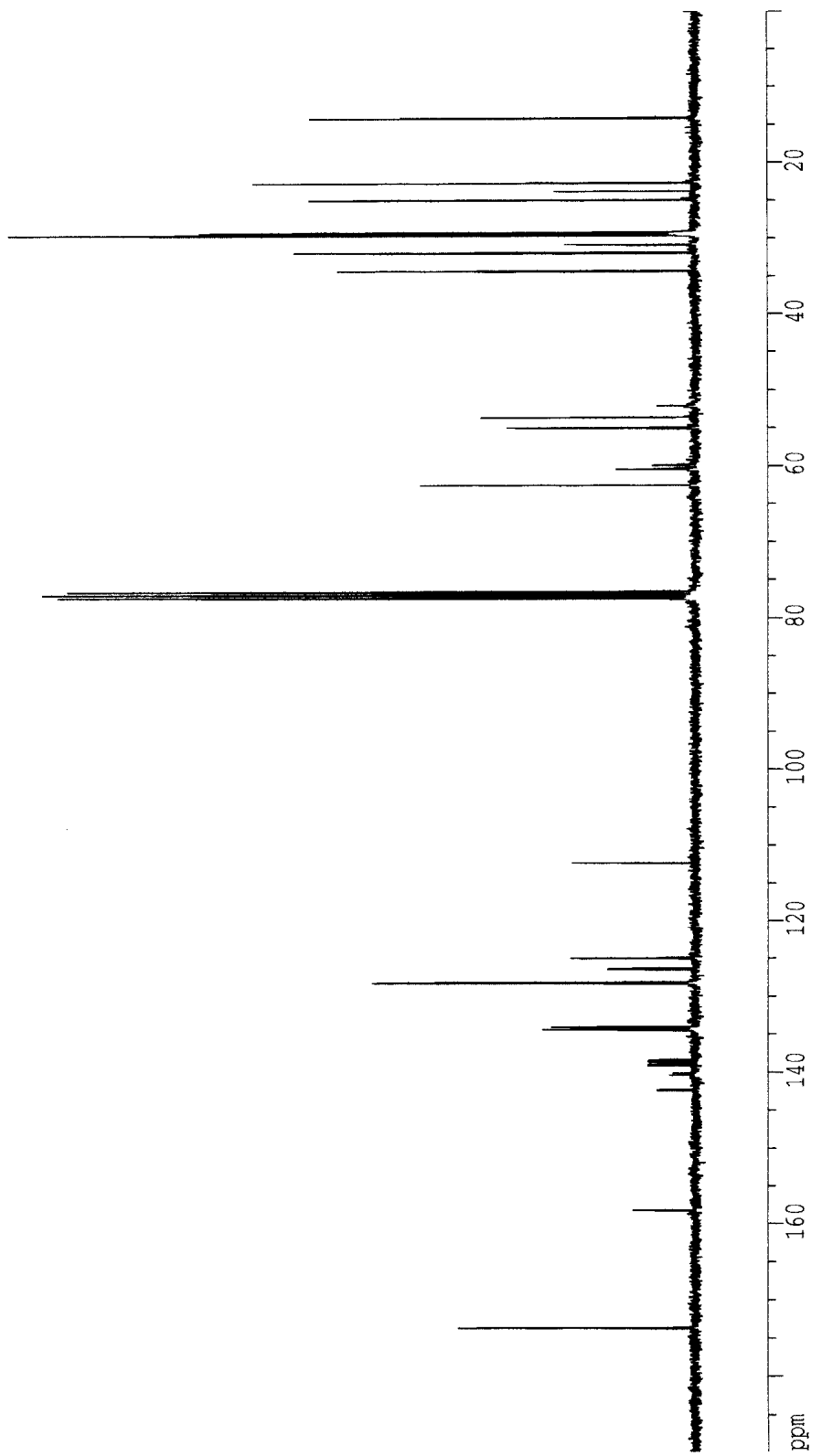


${ }^{31} \mathrm{P}$ NMR of 4d

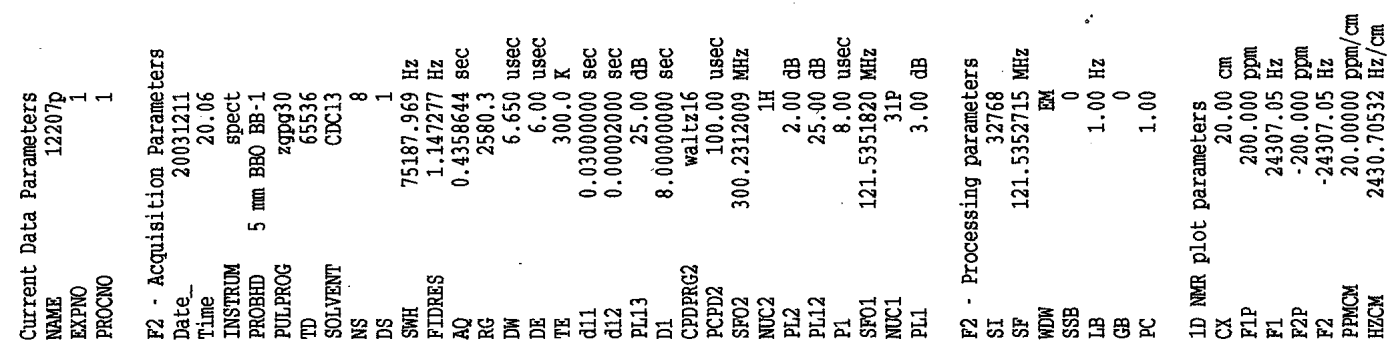

ธะ์.

แ̛đđ

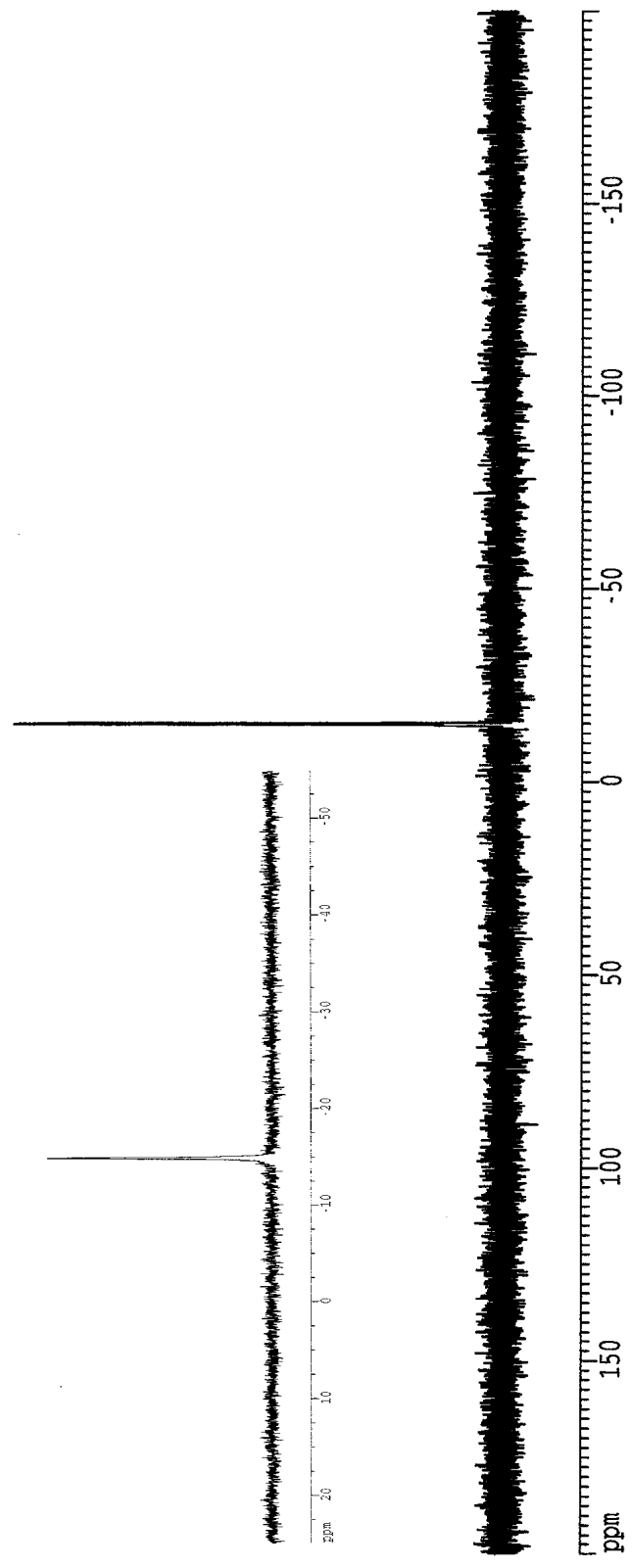


Chiral phase HPLC chart of 9a (Table 1, entry 14); 95\%ee

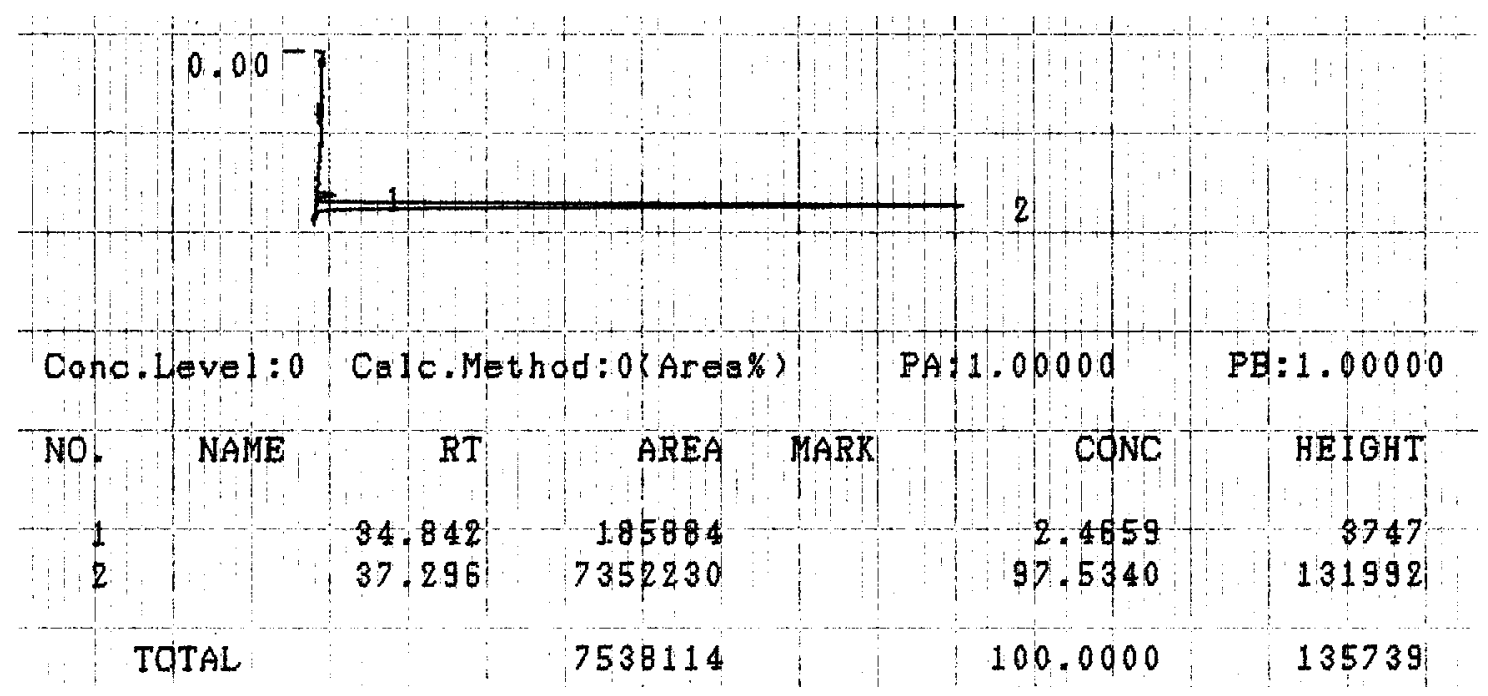

Daicel CHIRALCEL OD-H; Hexane $: i$-PrOH = $99: 1$; Flow $: 0.3 \mathrm{~mL} / \mathrm{min}$

Chiral phase HPLC chart of 9b (Table 2, entry 5); 97\%ee

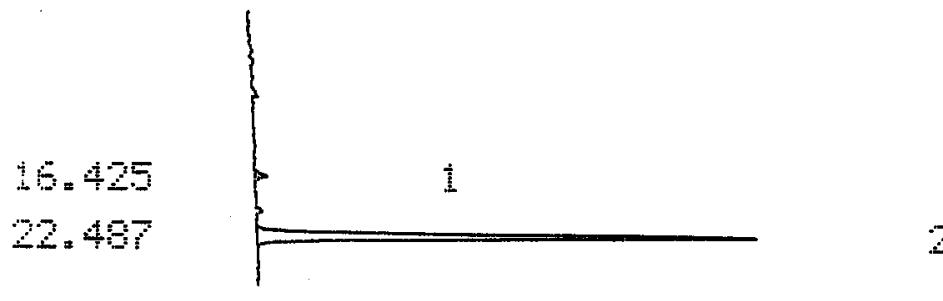

- \% CALCULATIN PEOULT -

TEST-DATA

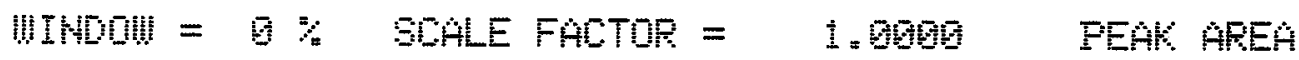

\begin{tabular}{|c|c|c|c|c|c|}
\hline FEAK & PThin & APEA & HEIGHT & mitic & AREA\% \\
\hline$\underline{1}$ & $16=25$ & 575 & 231 & & $i=6937$ \\
\hline 2 & 22.487 & 31203 & 9698 & & $90=9$ \\
\hline & TOTAL & $31790 \%$ & 9899 & & 100.6000 \\
\hline
\end{tabular}

Daicel CHIRALPAK AD-H; Hexane $: i$-PrOH $=95: 5$; Flow $: 1.0 \mathrm{~mL} / \mathrm{min}$ 
Chiral phase HPLC chart of 9c (Table 2, entry 3); 81\%ee

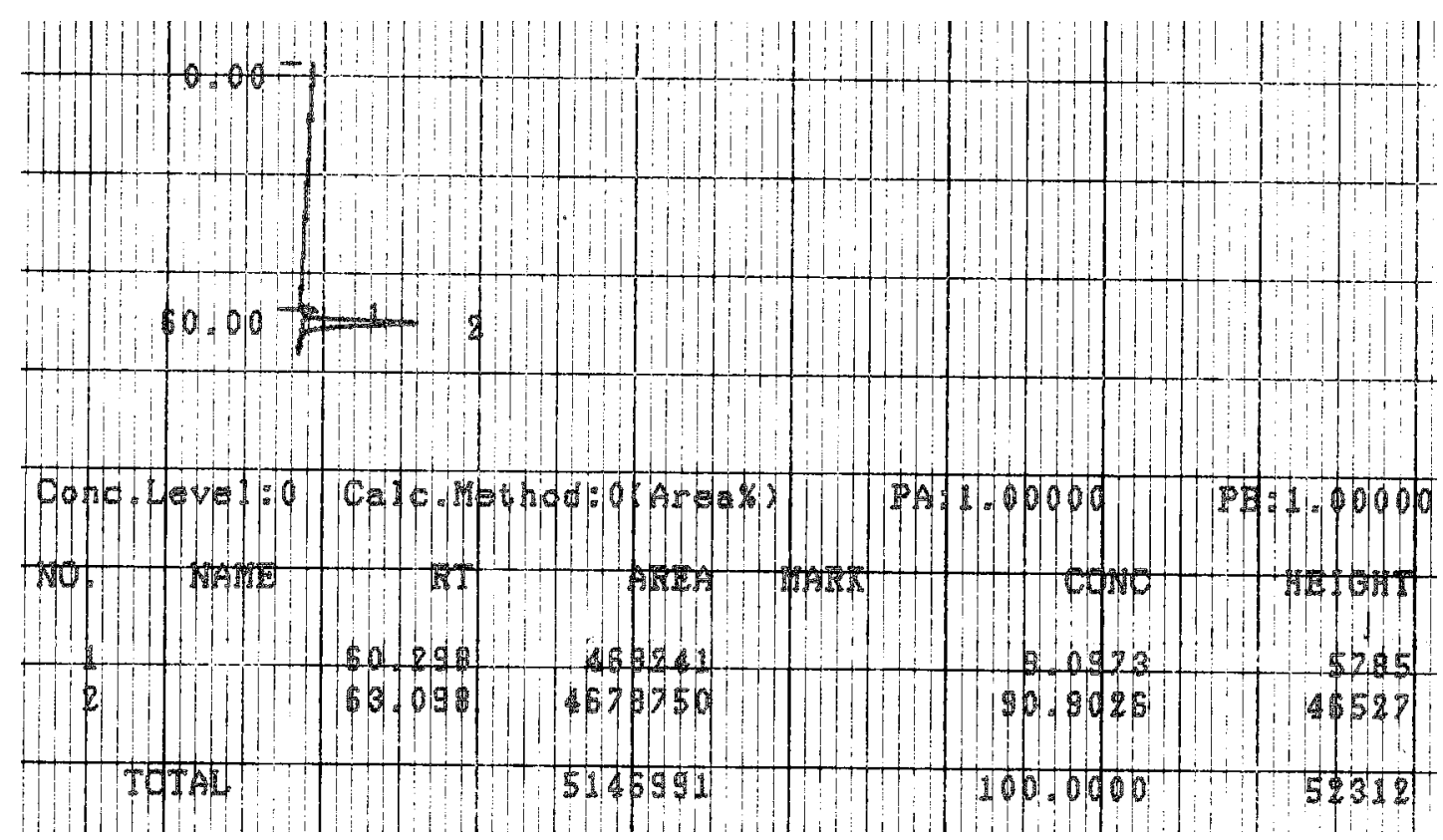

Daicel CHIRALCEL OD-H+OD at $0{ }^{\circ} \mathrm{C}$; Hexane $: i-\mathrm{PrOH}=199: 1$; Flow $: 0.3 \mathrm{~mL} / \mathrm{min}$ 